\title{
WHOLE EARTH TELESCOPE OBSERVATIONS OF THE WHITE DWARF G29-38: PHASE VARIATIONS OF THE 615 SECOND PERIOD
}

\author{
D. E. Winget, ${ }^{1,2,3}$ R. E. Nather, ${ }^{1}$ J. C. Clemens, ${ }^{1,3}$ J. Provencal, ${ }^{1}$ S. J. Kleinman, ${ }^{1}$ P. A. Bradley, ${ }^{1}$ \\ M. A. Wood ${ }^{1}$ C. F. Claver, ${ }^{1}$ E. L. Robinson, ${ }^{1}$ A. D. Grauer, ${ }^{4,5}$ B. P. Hine, ${ }^{6}$ G. Fontaine, ${ }^{3,7}$ \\ N. Achilleos, ${ }^{8}$ T. M. K. Marar, ${ }^{9}$ S. Seetha, ${ }^{9}$ B. N. Ashoka, ${ }^{9}$ D. O'Donoghue,${ }^{10}$ \\ B. Warner, ${ }^{10}$ D. W. Kurtz,${ }^{10}$ P. Martinez, ${ }^{10}$ G. Vauclair, ${ }^{11}$ M. CheVreton, ${ }^{12}$ \\ A. Kanaan, ${ }^{13}$ S. O. Kepler, ${ }^{13}$ T. Augusteijn, ${ }^{14}$ J. Van Paradijs, ${ }^{15}$ \\ C. J. HANSEN, ${ }^{16}$ AND JAMES LIEBERT ${ }^{17}$ \\ Received 1989 August 2; accepted 1990 January 15
}

\begin{abstract}
Using an extensive set of high-speed photometric observations obtained with the Whole Earth Telescope network, we show that the complex light curve of the ZZ Ceti (DAV) star G29-38 is dominated by a single, constant amplitude period of $615 \mathrm{~s}$ during the time span of our observations. The pulse arrival times for this period exhibit a systematic variation in phase readily explained by light-travel time effects produced by reflex orbital motion about an unseen companion. Our best-fit model to the observations indicates a highly eccentric orbit, a period of $109 \pm 13$ days and a minimum mass of $0.5 M_{\odot}$ for the companion.

Radial velocity variations predicted by this model are not observed, however, nor are these phase variations seen in another independent pulsation, so the origin of the phase variation remains a mystery. Any model involving intrinsic pulsation mechanisms must explain the large $(\sim 200 \mathrm{~s})$ phase change with no corresponding change in pulsation amplitude, and it shape, which mimics quite exactly the effects of binary orbital motion.
\end{abstract}

Subject headings: stars: binaries — stars: individual (G29-38) — stars: pulsation — stars: white dwarfs

\section{INTRODUCTION}

The star G29-38 (WD 2326+0.49, EG 159, ZZ Psc, LTT 16907 ) is a member of the class of pulsating white dwarf stars known as the ZZ Ceti (or DAV) stars, which display intrinsic luminosity variations of a few percent and periods ranging between 100 and $1000 \mathrm{~s}$. It is a particularly high amplitude example of the stars in this class, and like most of the highamplitude pulsators it has an extremely complex light curve (McGraw and Robinson 1975). Resolving this light curve into its component frequencies is of great interest from the standpoint of seismological investigations of white dwarf stars. The rich spectrum of observed modes, once resolved, can be compared with the spectrum of possible modes, allowing precise measurement of the stellar mass, and the mass of the surface layers. Individual modes can be monitored for amplitude stability to look for possible nonlinear effects and for long-term

\footnotetext{
${ }^{1}$ Department of Astronomy and McDonald Observatory, the University of Texas.

${ }^{2}$ Alfred P. Sloan Research Fellow.

3 Visiting Astronomer, Canada-France-Hawaii Telescope, operated by the National Research Council of Canada, Centre National de la Recherche Scientifique de France, and the University of Hawaii.

${ }^{4}$ Department of Physics and Astronomy, University of Arkansas at Little Rock.

5 Visiting Astronomer, Kitt Peak National Observatory.

6 NASA Ames Research Center.

${ }^{7}$ Departement de Physique, Université de Montréal, Canada.

${ }^{8}$ Australian National University, Canberra, Australia.

${ }^{9}$ Indian Space Research Organization, Bangalore, India.

10 Department of Astronomy, University of Cape Town, South Africa.

11 Observatoire Midi-Pyrenees, France.

12 Observatoire de Meudon, France.

13 Instituto de Fisica, Universidade Federal do Rio Grande do Sul, Brazil.

14 European Southern Observatory, Chile.

${ }^{15}$ Universiteit van Amsterdam, The Netherlands.

16 J.I.L.A., University of Colorado.

17 Steward Observatory, University of Arizona.
}

phase stability to study the evolution of the star (see Winget 1988 for a recent review of white dwarf seismology).

Quite apart from seismological motivations, recent infrared observations have generated a great deal of interest in G29-38. Zuckerman and Becklin $(1987 a, b)$ suggested that an observed IR excess might be due to the presence of a cool brown dwarf companion. Subsequent infrared spectroscopic observations (Tokunaga et al. 1988; Lester 1989) indicated that perhaps the apparent excess was due to the presence of a dust cloud, leaving the existence of an unseen companion in doubt.

Greenstein (1988) suggested that one could test for the presence of a possible brown dwarf companion by isolating a single stable frequency in the complex light curve of G29-38 and searching for modulations in its arrival time induced by light-travel time delays across the orbit. This technique is remarkably sensitive, as we have demonstrated in the cases of G117-B15A (Kepler et al. 1989), and L19-2 (O'Donoghue and Warner 1987). So far no significant variations have been detected in either object, to a limit of $\sim 1-2$ s over a time span of more than $10 \mathrm{yr}$. Had a planet of Jupiter's mass been present orbiting either star at the distance of Jupiter from the Sun, we would have found it.

The principal obstacle to the application of this time-lag technique lies in the complexity of the light curve of G29-38. Early observations of the object revealed power spectra which did not repeat from night to night and suggested that the light curve might be intrinsically unstable at a level sufficient to make its analysis at best difficult, and from a single site impossible (McGraw and Robinson 1975). We removed this latter obstacle by using the Whole Earth Telescope (WET), our global network of observatories (Nather 1989), to measure G29-38 essentially continuously for 2 weeks in 1988 November.

Comparison of a representative sample of the light curve, 


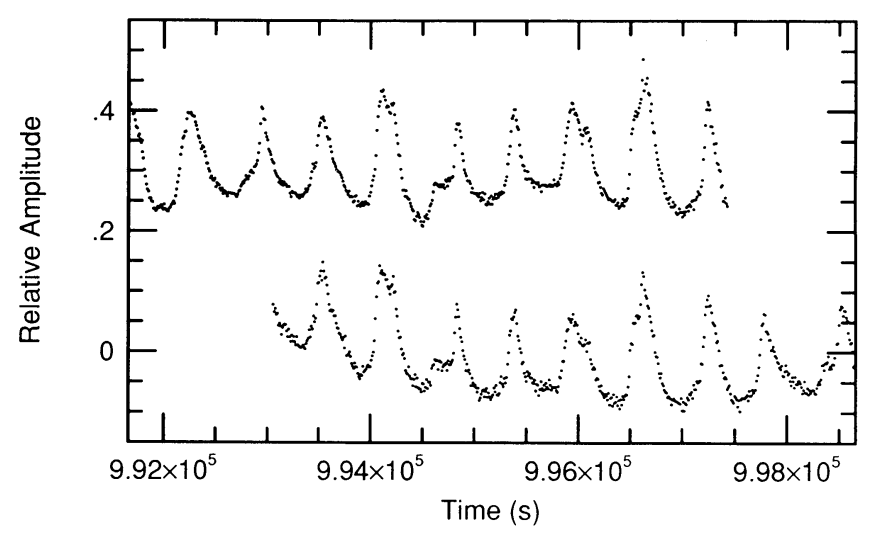

Fig. 1.-A portion of the light curve of G29-38, showing overlapping timeseries data obtained from Texas (upper curve) and the Hawaii (lower curve).

shown in Figure 1, with archival data (e.g., McGraw and Robinson 1975) indicates that the light curve has changed gradually over the years, in the direction of simplification. The amplitude spectrum (the square root of the power spectrum) bears this out: the spectrum of our complete data set, covering the region of greatest pulsation amplitude, is shown in Figure 2. Such changes are unimportant in the analysis of the phase variations of any individual period, or mode, if the amplitude of that mode is constant during the interval of interest; as long as this condition is met, linear pulsation theory describes the mode as a perfectly reliable clock (see discussion Kepler, Robinson, and Nather 1983).

The rich data set we obtained allowed us to resolve completely the dominant $615 \mathrm{~s}(1626 \mu \mathrm{Hz})$ peak in the spectrum and to demonstrate its freedom from beating or other interactions with other periodicities, during a time interval of $\sim 7 \times 10^{6} \mathrm{~s}$.

Data obtained from McDonald Observatory prior to the WET run served as a point of comparison, and real-time data analysis during the run allowed us to isolate the $615 \mathrm{~s}$ period and test it for stability. Our original plan was to concentrate on our second target once the G29-38 light curve was resolved, but when we detected significant curvature in the time-lag $(O-C)$ stability diagram we readjusted our observing priorities to include both objects.

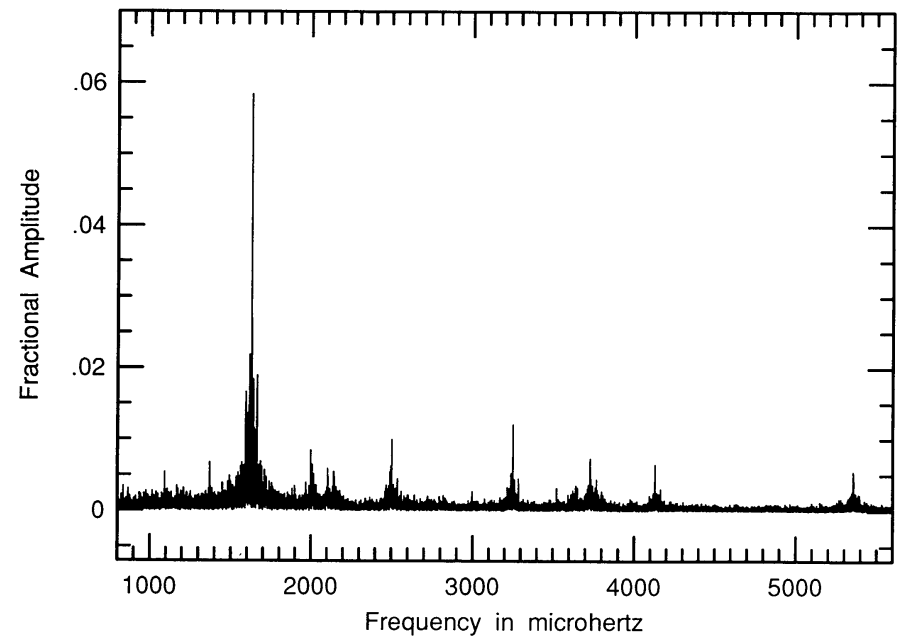

FIG. 2.-A portion of the amplitude spectrum of G29-38, showing the region of highest pulsation amplitudes.
After the WET run we continued to observe G29-38 whenever possible from several of our sites, until we lost it in the Sun (after early 1989 January). When we regained it from the Sun in June, the $615 \mathrm{~s}$ period had begun to change significantly in amplitude, so this mode could no longer be relied upon to constitute a reliable clock. Below we present the phase variations we observed in the $615 \mathrm{~s}$ period over the interval (1988 October-1989 January) during which its amplitude remained unchanged.

\section{HIGH-SPEED PHOTOMETRIC OBSERVATIONS}

In order to look for variations in pulse arrival times we must first isolate an individual periodicity in the Fourier transform of a light curve, unblended with power from nearby frequencies. To do this we require a data set with a total time span greater than $T$, and ideally greater than $2 T$, where $T \equiv 1 / \delta v$, and $\delta v$ is the frequency separation between the frequency of interest and that of its nearest neighbor. For the DAV stars $T$ can be as large as several days (see Winget 1988 and references therein). Further, we need essentially continuous data (i.e., without significant gaps) so that aliases and spectral leakage effects in the Fourier transform are not hopelessly confused with actual power. Based on our experience with other DAV stars, we require uninterrupted measurements during about five rotations of our planet.

This is a one-time requirement, however. Once we have resolved the light curve, we can select a suitable period such that shorter, single-site measurements will isolate it from its nearest neighbors and allow us to monitor its stability. We can, if need be, combine several runs, since alias peaks in the Fourier transform can be identified and discounted. After suitable high-resolution analysis, we found that data taken both before and after the WET run could be used to search for variations in pulse arrival times.

Our total high-speed photometric data base is summarized in the journal of observations in Table 1. The observations span the period from late October of 1988 to mid-January of 1989 and are listed chronologically by run start time in coordinated universal time (UTC). Observations were made without a filter to maximize the photon counting rate. Because we used photomultiplier tubes with blue-sensitive bi-alkali photocathodes, and because the target star is very blue, our effective spectral bandpass was roughly that of a Johnson $B$ filter.

Two sites, in France and Australia, used three-channel photometers, continuously monitoring the sky as well as the target and comparison star. All other sites employed two-star photometers, which measured the program star and a comparison (to ensure photometric conditions), but interrupted their observations roughly every 30 to 60 minutes for sky measurements, depending on sky brightness. The South African photometer had its second (comparison star) channel disabled, preventing measurements of the comparison star. Sky measurements typically lasted $\sim 1$ minute and were taken near the flat minima in the light curve to make it possible to interpolate the data for these short intervals. Observers took pains to take these sky measurements at irregular intervals to avoid introducing artifacts into the Fourier transform.

\section{ANALYSIS OF THE 615 SECOND PERIOD}

We reduced each run from an individual site-usually within minutes after the run was finished, thanks to global electronic mail-using the techniques described in Kepler et al. 
TABLE 1

\begin{tabular}{|c|c|c|c|c|c|}
\hline Run Name & Observatory & $\begin{array}{l}\text { Aperture } \\
(\mathrm{m})\end{array}$ & $\begin{array}{l}\text { Date } \\
\text { (UT) }\end{array}$ & $\begin{array}{l}\text { Start } \\
\text { (UT) }\end{array}$ & $\begin{array}{c}\text { Length } \\
\text { (s) }\end{array}$ \\
\hline JCC- 0055 & McDonald & 0.80 & 1988 Oct 24 & $2: 00: 00$ & 9800 \\
\hline JCC-0057 …....... & McDonald & 0.80 & 1988 Oct 25 & $1: 50: 00$ & 13650 \\
\hline JCC-0059 ........ & McDonald & 0.80 & 1988 Oct 26 & $4: 50: 00$ & 11910 \\
\hline SAO-4455 ...... & SAAO & 0.75 & 1988 Nov 4 & $18: 38: 00$ & 20100 \\
\hline MAW-0017 ...... & McDonald & 2.10 & 1988 Nov 6 & $1: 42: 20$ & 18420 \\
\hline SAO-4455 ....... & SAAO & 0.75 & 1988 Nov 6 & $18: 23: 00$ & 10000 \\
\hline MAW-0019 ..... & McDonald & 2.10 & 1988 Nov 7 & $1: 31: 00$ & 24860 \\
\hline REN-0040 ...... & Siding Spring & 1.00 & 1988 Nov 7 & $11: 12: 30$ & 9600 \\
\hline ADG-0085 ....... & Mauna Kea & 0.60 & 1988 Nov 8 & $7: 51: 00$ & 6980 \\
\hline REN-0042 …... & Siding Spring & 1.00 & 1988 Nov 8 & 9:41:00 & 18700 \\
\hline MAW-0023 ...... & McDonald & 2.10 & 1988 Nov 9 & $1: 21: 10$ & 10750 \\
\hline MAW-0024 ...... & McDonald & 2.10 & 1988 Nov 9 & $4: 54: 40$ & 13020 \\
\hline REN-0044 ...... & Siding Spring & 1.00 & 1988 Nov 9 & $9: 41: 10$ & 10410 \\
\hline SAO-4456 ….... & SAAO & 0.75 & 1988 Nov 9 & $18: 53: 30$ & 18900 \\
\hline MAW-0026 ..... & McDonald & 2.10 & 1988 Nov 10 & $1: 23: 00$ & 27140 \\
\hline REN-0045 …... & Siding Spring & 1.00 & 1988 Nov 10 & $9: 28: 50$ & 21100 \\
\hline SAO-4457 $\ldots \ldots \ldots$ & SAAO & 0.75 & 1988 Nov 10 & $18: 33: 50$ & 19200 \\
\hline GV-0037 ......... & OHP & 1.93 & 1988 Nov 11 & $18: 59: 20$ & 5390 \\
\hline MAW-0029 ...... & McDonald & 2.10 & 1988 Nov 12 & $1: 24: 10$ & 8330 \\
\hline CFC-0002 $\ldots \ldots$. & McDonald & 0.90 & 1988 Nov 12 & $3: 41: 01$ & 13500 \\
\hline REN-0049 ...... & Siding Spring & 1.00 & 1988 Nov 12 & $9: 30: 10$ & 15650 \\
\hline SAO-4458A ...... & SAAO & 0.75 & 1988 Nov 12 & $19: 41: 53$ & 4900 \\
\hline MAW-0031 ...... & McDonald & 2.10 & 1988 Nov 13 & $0: 51: 20$ & 8300 \\
\hline CFC-0005 ...... & McDonald & 0.90 & 1988 Nov 13 & $3: 03: 50$ & 15500 \\
\hline REN-0053 ....... & Siding Spring & 1.00 & 1988 Nov 13 & $9: 28: 20$ & 8080 \\
\hline GV-0039 ......... & OHP & 1.93 & 1988 Nov 13 & $18: 12: 30$ & 7690 \\
\hline CFC-0009 ....... & McDonald & 0.90 & 1988 Nov 14 & $3: 22: 30$ & 14150 \\
\hline CFC-0010 ........ & McDonald & 0.90 & 1988 Nov 15 & $3: 46: 30$ & 10570 \\
\hline MAW-0036 ...... & McDonald & 2.10 & 1988 Nov 16 & $1: 07: 20$ & 23780 \\
\hline ADG-0097 ........ & Mauna Kea & 0.60 & 1988 Nov 16 & $6: 28: 00$ & 18640 \\
\hline MAW-0038 ...... & McDonald & 2.10 & 1988 Nov 17 & $0: 58: 20$ & 4920 \\
\hline SAO-4466 $\ldots \ldots \ldots$ & SAAO & 0.75 & 1988 Nov 17 & $18: 43: 00$ & 10000 \\
\hline MAW-0040 ....... & McDonald & 2.10 & 1988 Nov 18 & $6: 05: 40$ & 7130 \\
\hline SAO-4468 $\ldots \ldots \ldots$ & SAAO & 0.75 & 1988 Nov 18 & $18: 43: 00$ & 3700 \\
\hline MAW-0042 ...... & McDonald & 2.10 & 1988 Nov 19 & $0: 52: 20$ & 23130 \\
\hline MLF-0001 ........ & McDonald & 0.80 & 1988 Nov 20 & $1: 19: 28$ & 19520 \\
\hline SAO-4472A ...... & SAAO & 0.75 & 1988 Nov 21 & $18: 52: 00$ & 8000 \\
\hline MLF-0003 ....... & McDonald & 0.80 & 1988 Nov 22 & $1: 04: 03$ & 18350 \\
\hline SJK-0005 ….... & McDonald & 0.80 & 1988 Nov 24 & $1: 29: 40$ & 21330 \\
\hline CFC-0016 $\ldots \ldots \ldots$ & McDonald & 0.80 & 1988 Nov 26 & $1: 01: 40$ & 7710 \\
\hline CFC-0018 ….... & McDonald & 0.80 & 1988 Nov 28 & $0: 57: 50$ & 22040 \\
\hline PAB-0002 …..... & McDonald & 0.80 & 1988 Nov 29 & $1: 14: 50$ & 19510 \\
\hline PAB-0004 ......... & McDonald & 0.90 & 1988 Dec 1 & $0: 44: 30$ & 18630 \\
\hline JCC-0063 ….... & Mauna Kea CFHT & 3.60 & 1988 Dec 1 & $5: 54: 30$ & 8010 \\
\hline JCC-0066 ${ }^{\mathrm{a}} \ldots \ldots$ & Mauna Kea CFHT & 3.60 & 1988 Dec 2 & $4: 59: 10$ & 8650 \\
\hline PAB-0009 …...... & McDonald & 0.90 & 1988 Dec 3 & $0: 54: 10$ & 17510 \\
\hline PAB-0011 ........ & McDonald & 0.90 & 1988 Dec 4 & $0: 56: 20$ & 16900 \\
\hline MLF-0004 ........ & McDonald & 0.90 & 1988 Dec 14 & $1: 21: 21$ & 12140 \\
\hline MLF-0005 ........ & McDonald & 0.90 & 1988 Dec 15 & $1: 17: 04$ & 6170 \\
\hline JCC-OO69 ...... & McDonald & 0.90 & 1988 Dec 21 & $1: 25: 00$ & 10890 \\
\hline JCC-0071 & McDonald & 0.90 & 1988 Dec 22 & $1: 24: 00$ & 8090 \\
\hline ADG-0100 ....... & KPNO & 1.30 & 1988 Dec 30 & $2: 4700$ & 9500 \\
\hline ADG-0104 ....... & KPNO & 1.30 & 1988 Dec 31 & $1: 57: 00$ & 12860 \\
\hline ADG-0112 ........ & KPNO & 1.30 & 1989 Jan 6 & $1: 49: 00$ & 11350 \\
\hline ADG-0118 ....... & KPNO & 1.30 & 1989 Jan 9 & $1: 56: 00$ & 10380 \\
\hline
\end{tabular}

a $5 \mathrm{~s}$ integration. All others are $10 \mathrm{~s}$.

(1982). First, we subtracted sky, then normalized each run by dividing by a polynomial of up to third order. At this point if the run was interrupted by cloud or other problems for longer than $\sim 100 \mathrm{~s}$, we broke it up into parts, discarding the bad data ponts. We treated each segment-before and after the interruption - as a separate run. In order to reduce the amplitude of the sidelobes in the Fourier transform, we tapered the first and last $10 \%$ of each run with a cosine-bell taper. Thus our data sets consist of fractional intensity as a function of time. We note that these and all transforms in this paper are amplitude spectra - the square root of the (more traditional) power spectra-with the vertical scale corresponding to fractional semi-amplitude. The amplitudes have been adjusted to account for the taper.

As each new data set came in, we put it on a uniform time base by converting the times in UTC to Julian Ephemeris Time (taking appropriate count of leap seconds). Next we computed the barycentric correction - using the prescription and coeffi- 
cients from the Astronomical Almanacs for 1988 and 1989 for the start of the run and applied it to all the times, which were then kept in running seconds with respect to our arbitrarily chosen zero epoch (corresponding to the run start of the first WET run, s4453) of Barycentric Julian Dynamical Date $2,447,470.280774$. We concatenated each new data set to the previous data and computed an updated Fourier transform.

In Figure 1 we display a typical portion of the light curve where overlapping data from two sites were obtained. We routinely allowed small overlaps of 5-10 minutes when possible to ensure continuity in the data set; we occasionally obtained larger overlaps, such as the one shown, to monitor the reproducibility of the light curve from site to site. Although obtained at very different air masses, the match of overlapping data shown in Figure 1 is completely satisfactory.

A full day elapsed between the first data from South Africa and the start of the WET run at the other sites; however, after 2 full days of operation, the 1 day aliases introduced by this initial gap were completely gone. Further, all of the individual light curves, and their transforms, were dominated by one large amplitude period at $615 \mathrm{~s}$. As the data accumulated, it became increasingly apparent that this period was an isolated peak, at least during the span of our observations, and so we chose it to analyze. As it later became apparent, this was the only stable peak with sufficiently large amplitude for a quick-look analysis.

We show in Figure 3 that portion of the transform surrounding the $615 \mathrm{~s}$ period. We show in vertical mirror image what we call the window of the transform: the transform of a single sine curve of constant amplitude sampled and tapered in the same way as the observations. Close inspection of the data transform reveals that there are no significant aliasing ambiguities due to gaps in the data and that the transform is indistinguishable from the transform of a single sine curve in the vicinity of the $615 \mathrm{~s}$ peak. Any adjacent periodicity, to remain concealed, would have to lie within $1 \mu \mathrm{Hz}$ of the observed frequency - a limit already finer than any known splitting observed in a compact pulsator.

The amplitude of this peak remained constant in the transform as we continued to accumulate data, further indicating that there were no closely spaced periods which remained

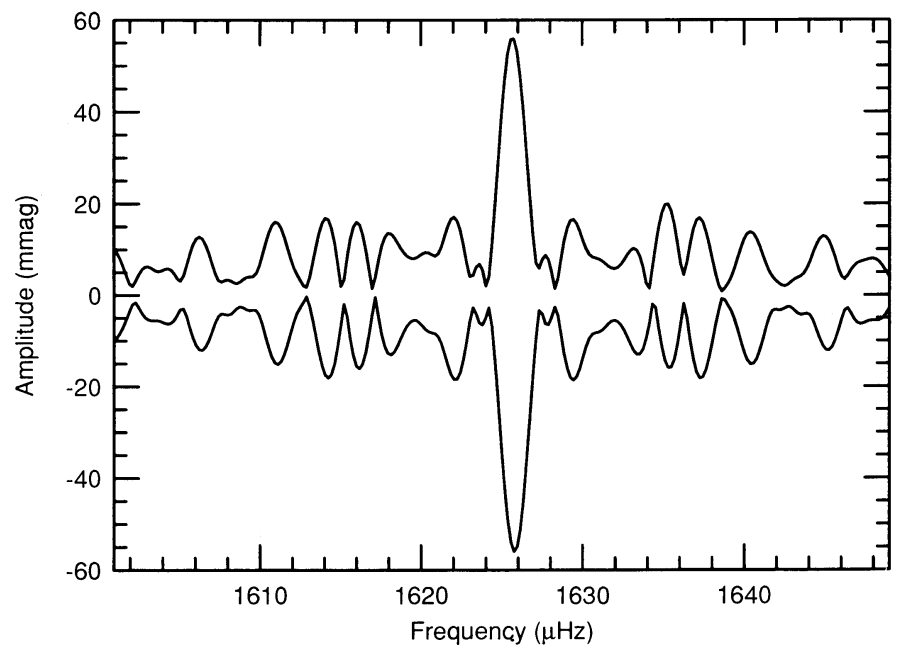

FIG. 3.-The amplitude spectrum of the $615.15 \mathrm{~s}$ periodicity (upper curve) and its spectral window (lower curve, vertically reversed), for the timeconcentrated data only.

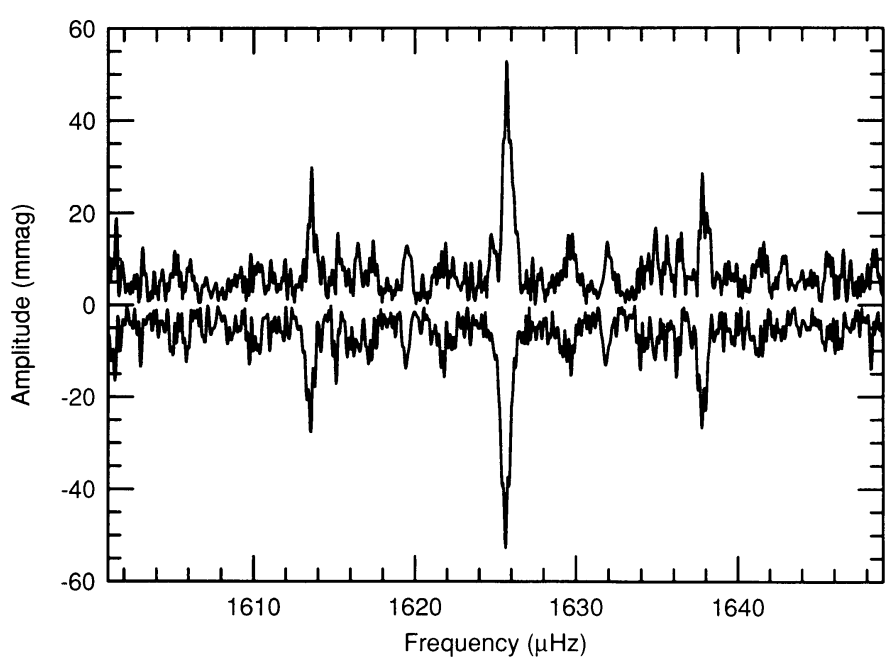

FIG. 4.-The amplitude spectrum of the $615.15 \mathrm{~s}$ periodicity (upper curve) and its spectral window (lower curve, vertically reversed), for all of the data on G29-38.

unresolved. This point is further strengthened by Figure 4, which is a portion of the transform of the entire dataset: the amplitude of the $615 \mathrm{~s}$ peak is the same as in Figure 3. Although other portions of the spectrum seemed not to mirror this stability, at least over this data set the amplitude of the 615 s period remained constant to within our ability to measure it. Note that $24 \mathrm{hr}$ aliases crept back into the transform in Figure 4 because the follow-up observations, as well as the October data, were all taken from the same longitude. This introduces no ambiguities in the analysis because the correct frequency was determined from the WET data.

Again in Figure 4 the $615 \mathrm{~s}$ mode is indistinguishable from the window (the small peak to the left of the $615 \mathrm{~s}$ peak is an artifact of moonrise, discussed below). This tightens the constraint on the existence of any significant peaks near the $615 \mathrm{~s}$ period: any concealed peaks would have to be closer than 0.1 $\mu \mathrm{Hz}$. Having demonstrated that this was a single peak, stable in amplitude over at least the span of our data, $7 \times 10^{6} \mathrm{~s}$, our next step was to investigate the pulse arrival times.

\section{THE $(O-C)$ DIAGRAM FOR THE 615.15 SECOND PERIOD}

In order to search for variations in the pulse arrival time we constructed an $(O-C)$ diagram. Our first step was to establish the best value for the $615 \mathrm{~s}$ period and to construct an ephemeris for the pulse arrival times based on that period, giving us the calculated time of arrival. We used the value of the period from the Fourier transform, $615.15 \mathrm{~s}$.

The data shown in Figures 3 and 4 demonstrated that the $615 \mathrm{~s}$ period could be distinguished from all other frequencies in any data set over $\sim 2 \mathrm{hr}$ in length, so we could compute a best time of maximum from each run individually. When we subtract from this observed time of maximum the expected time of maximum from the calculated ephemeris, we have an $(O-C)$ value in seconds.

We show in Table 2 a list of the times of maxima, and the formal errors of fit, for the $615.15 \mathrm{~s}$ period for each run. We obtained each time of maximum and amplitude by fitting our nominal period of $615.15 \mathrm{~s}$ to each run, using a least-squares technique. As is common in sinusoidal fits in the presence of scintillation noise and competing periodicities, the formal 
TABLE 2

$O-C$ DATA

\begin{tabular}{|c|c|c|c|}
\hline Run Name & $\begin{array}{l}T_{\max } \\
\text { (s) }\end{array}$ & $\begin{array}{c}O-C \\
\text { (s) }\end{array}$ & $\underset{\text { (mmag) }}{\text { Amp }}$ \\
\hline JCC-0055 & $-1,010,107 \pm 3$ & -100 & $65 \pm 2$ \\
\hline JCC-0057 & $-923,993 \pm 2$ & -107 & $63 \pm 1$ \\
\hline JCC-0059 & $-826,797 \pm 3$ & -101 & $55 \pm 2$ \\
\hline SAO-4453 & $89 \pm 2$ & 0 & $53 \pm 2$ \\
\hline MAW-0017 & $112,018 \pm 2$ & -3 & $56 \pm 1$ \\
\hline SAO-4455 .. & $172,317 \pm 3$ & 6 & $57 \pm 2$ \\
\hline MAW-0019 ...... & $198,144 \pm 2$ & -3 & $48 \pm 1$ \\
\hline REN-0040 $\ldots \ldots \ldots$ & $232,566 \pm 3$ & -30 & $54 \pm 1$ \\
\hline ADG-0085 $\ldots \ldots \ldots$ & $307,037 \pm 5$ & 8 & $52 \pm 3$ \\
\hline REN-0042 ....... & $313,782 \pm 2$ & -14 & $59 \pm 1$ \\
\hline MAW-0023 ...... & $369,793 \pm 3$ & 19 & $44 \pm 2$ \\
\hline MAW-0024 ....... & $382,709 \pm 3$ & 17 & $58 \pm 1$ \\
\hline REN-0044 & $401,139 \pm 3$ & -8 & $43 \pm 3$ \\
\hline SAO-4456 & $433,116 \pm 2$ & -19 & $59 \pm 1$ \\
\hline MAW-0026 ....... & $456,505 \pm 2$ & -5 & $52 \pm 1$ \\
\hline REN-0045. & $485,427 \pm 2$ & 5 & $53 \pm 1$ \\
\hline SAO-4457. & $518,636 \pm 2$ & -4 & $65 \pm 1$ \\
\hline GV-0037 & $606,606 \pm 3$ & -1 & $63 \pm 2$ \\
\hline MAW-0029 & $629,365 \pm 3$ & -2 & $58 \pm 2$ \\
\hline CFC-0002 _........ & $637,277 \pm 2$ & -18 & $63 \pm 1$ \\
\hline REN-0049 & $658,872 \pm 2$ & -23 & $57 \pm 1$ \\
\hline SAO-4458A ...... & $695,157 \pm 4$ & -31 & $60 \pm 2$ \\
\hline MAW-0031 ...... & $713,612 \pm 4$ & -31 & $52 \pm 2$ \\
\hline CFC-0005 ......... & $721,616 \pm 2$ & -24 & $49 \pm 1$ \\
\hline REN-0053 ........ & $745,002 \pm 3$ & -14 & $55 \pm 2$ \\
\hline GV-0039 .. & $776,357 \pm 3$ & -31 & $65 \pm 2$ \\
\hline CFC-0009 & $809,567 \pm 2$ & -40 & $58 \pm 1$ \\
\hline CFC-0010 & $896,909 \pm 3$ & -49 & $64 \pm 2$ \\
\hline MAW-0036 & $973,822 \pm 2$ & -29 & $66 \pm 1$ \\
\hline ADG-0097 ….... & $993,483 \pm 3$ & -53 & $69 \pm 2$ \\
\hline MAW-0038 ....... & $1,059,925 \pm 5$ & -47 & $57 \pm 2$ \\
\hline SAO-4466 ........ & $1,123,839 \pm 3$ & -42 & $62 \pm 2$ \\
\hline MAW-0040 ...... & $1,164,492 \pm 3$ & -56 & $67 \pm 2$ \\
\hline SAO-4468 $\ldots \ldots \ldots$ & $1,210,028 \pm 7$ & -41 & $51 \pm 2$ \\
\hline MAW-0042 ...... & $1,232,148 \pm 3$ & -66 & $70 \pm 2$ \\
\hline MLF-0001 ........ & $1,320,078 \pm 3$ & -103 & $61 \pm 2$ \\
\hline SAO-4472A $\ldots \ldots$ & $1,469,596 \pm 3$ & -66 & $65 \pm 2$ \\
\hline MLF-0003 ........ & $1,492,333 \pm 2$ & -90 & $59 \pm 1$ \\
\hline SJK-0004 ….... & $1,580,919 \pm 2$ & -86 & $69 \pm 2$ \\
\hline SJK-0005 & $1,666,426 \pm 2$ & -84 & $59 \pm 1$ \\
\hline CFC-0016 ......... & $1,837,422 \pm 4$ & -100 & $61 \pm 3$ \\
\hline CFC-0018 $\ldots \ldots \ldots$ & $2,010,293 \pm 2$ & -86 & $48 \pm 1$ \\
\hline PAB-0002 ........ & $2,097,604 \pm 2$ & -126 & $55 \pm 1$ \\
\hline PAB-0004 …..... & $2,268,581 \pm 2$ & -161 & $58 \pm 2$ \\
\hline JCC-0063 $\ldots \ldots \ldots$ & $2,287,068 \pm 3$ & -129 & $65 \pm 2$ \\
\hline JCC-0066 ….... & $2,370,094 \pm 2$ & -148 & $40 \pm 1$ \\
\hline PAB-0009 ......... & $2,442,691 \pm 2$ & -139 & $60 \pm 1$ \\
\hline PAB-0011 ........ & $2,529,427 \pm 2$ & -138 & $63 \pm 1$ \\
\hline MLF-0004 ........ & $3,393,662 \pm 3$ & -189 & $50 \pm 2$ \\
\hline MLF-0005 ........ & $3,479,806 \pm 3$ & -166 & $68 \pm 2$ \\
\hline JCC-0069 ….... & $3,998,979 \pm 4$ & -179 & $40 \pm 2$ \\
\hline JCC-0071 & $4,085,076 \pm 5$ & -204 & $56 \pm 3$ \\
\hline ADG-0100 $\ldots \ldots \ldots$ & $4,781,082 \pm 2$ & -213 & $55 \pm 2$ \\
\hline ADG-0104 ........ & $4,867,808 \pm 3$ & -202 & $59 \pm 2$ \\
\hline ADG-0112 ....... & $5,382,410 \pm 2$ & -221 & $59 \pm 1$ \\
\hline ADG-0118 ........ & $5,642,021 \pm 3$ & -204 & $54 \pm 2$ \\
\hline
\end{tabular}

errors underestimate the true errors, indicated by the scatter, by a factor between 2 and 4; the relative error values, however, provide a means of comparing the quality of individual runs.

Our chosen epoch for phase comparison was the first run obtained with the WET, and showed immediately that the maxima were arriving $\sim 100 \mathrm{~s}$ later than during the preliminary runs in October, some 17 days earlier. Near the beginning of the WET run the arrival times continued to come later and later, and we assumed that we simply had the $615 \mathrm{~s}$ period slightly wrong, which would introduce a linear trend into the $(O-C)$ plot. As the WET run progressed, however, the arrival times gradually began to reverse this trend, curving downward on the $(O-C)$ plot as they arrived earlier and earlier, until they arrived $\sim 200$ s earlier than at our comparison epoch.

Even in the presence of the substantial phase scatter of order $10 \mathrm{~s}$, it was clear that there were large, long-term, systematic variations in the time of pulse arrival which demanded explanation.

\section{POSSIBLE INTRINSIC CAUSES OF THE PHASE VARIATIONS}

In general terms, there are three known ways, intrinsic to the pulsating star, of producing significant modulations of the phase of a pulsation mode: linear interference (i.e., beating) with other modes, nonlinear interactions with other modes (i.e. mode coupling), and rotation of the pulsation axis about the line of sight. These three types of modulations all have one thing in common: all of them produce amplitude modulation which occurs simultaneously with the phase modulation. If any of these perturbing processes is in operation, the net effects on phase should be unimportant over any particular interval in which the pulsation amplitude remains constant.

The first possibility we considered for the variations in the time-lag $(O-C)$ diagram was the beating of closely spaced modes. This effect has been observed in other DAV stars: in R548 (Stover et al. 1980), in G226-29 (Kepler et al. 1983), and in L19-2 (O'Donoghue and Warner 1987). The spacings between these modes yield beat periods on the order of days, implying stellar rotation rates of that same order if the frequency splitting is due to stellar rotation.

The nonradial $g$-mode spectrum is not rich enough to produce such closely spaced modes from integral steps in the spherical harmonic index, or radial overtone number; that spacing would separate periodicities by 10-20 s (Kawaler 1988), corresponding to beat periods of a few hours. However, rotation of the star breaks the degeneracy (in frequency) of nonradial modes with different azimuthal quantum numbers $m$ (see Cox 1980 or Unno et al. 1989). The beat period is then comparable to the rotation period of the star. This beating can potentially produce substantial phase variations in the blended peak if the individual frequencies are not resolved and the amplitude of the closely spaced modes are comparable. This situation occurs when the timings are obtained from runs that are short compared with the rotation period.

We could test this possibility with the data in hand. The Fourier transform of the total data set (Fig. 4) in the region of $615 \mathrm{~s}$ period shows a single peak. In order to modulate the phase of this peak, any unresolved modes must also modulate its amplitude on the same time scale, so we could set stringent limits by investigating the amplitude of the $615 \mathrm{~s}$ peak as a function of time.

We plotted the amplitude for each run from Table 2, as shown in Figure 5. There is no obvious trend on a time scale of $10^{7} \mathrm{~s}$, the time scale of the phase variations actually observed. Indeed if we use the least-squares technique to fit the data with a sinusoid of period $10^{7} \mathrm{~s}$, the amplitude in fractional intensity units is $0.002 \pm 0.003$. Therefore the amplitude of any possible unresolved mode must be less than $5.5 \%$ of the amplitude of the main peak. Such an amplitude variation can produce at most a 2-3 s phase variation-insignificant in comparison with the variation observed.

We do note that while there are no significant sinusoidal variations in our amplitude data, there is a local maximum 


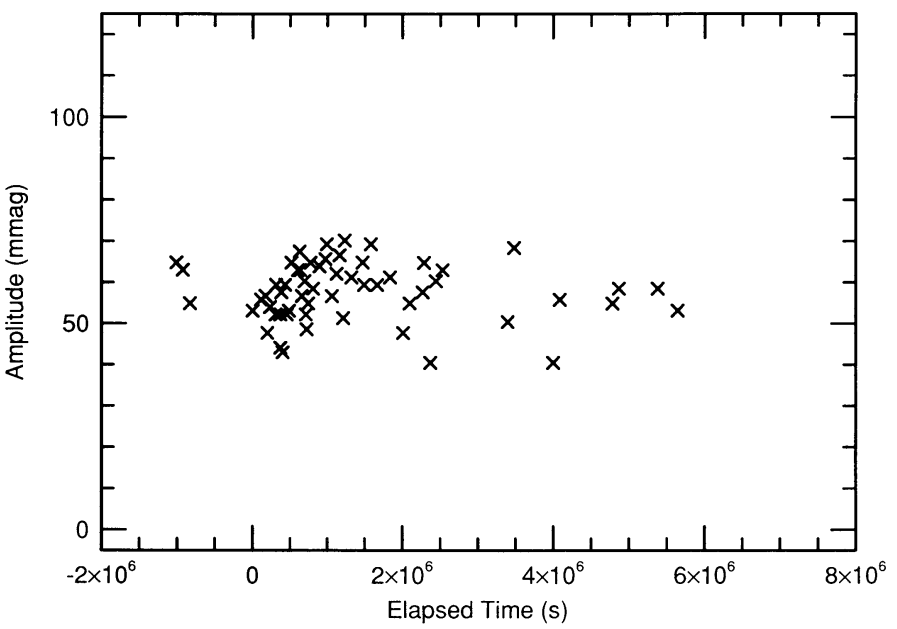

FIG. 5.-Individual amplitude measurements of the $615.15 \mathrm{~s}$ periodicity plotted against elapsed time.

near $t=1,230,000 \mathrm{~s}$. The maximum appears to be marginally significant, although at $15 \%$ it is smaller than the scatter. It is, however, an artifact, resulting in a systematic increase in pulsation amplitude for runs in which the local moonrise occurs during our observations at each site. The changing sky brightness was not modeled well enough in our reduction procedures where sky was not measured continuously; the effect is completely absent in the three-channel photometer data.

We next considered the second possible internal cause of phase modulations: nonlinear interactions between modes. Until about 1982, nonlinear effects were often suggested as a potential explanation for the night-to-night variations in the power spectra and the presence of harmonically related frequencies in many of the complex pulsators. The night-to-night variations have been shown, in every case investigated to date, to result from the beating of closely spaced modes (Winget 1988; Dziembowski 1988). Nonlinear coupling of modes remains, however, the best explanation for the presence of harmonically related frequencies, and so we considered phase variations they might cause.

The complete stability in amplitude of the $615 \mathrm{~s}$ variations also eliminated the possibility of nonlinear mode interactions as an explanation for the observed phase variations: any nonlinear effect which has such a dramatic influence on the phase must also influence the amplitude on the same time-scale as the phase variations. Nonlinear mode-coupling produces changes in the power spectra by changing the energy in two or more modes, transferring energy from one mode to another in a periodic, quasi-periodic, or even chaotic fashion. The energy is proportional to the square of the amplitude, thus energy transferred in this manner should also cause changes in the amplitude (Dziembowski 1988; Buchler 1988).

Finally, we considered the possibility of phase modulations resulting from the changes in the angle between the pulsation axis and the line of sight. This can arise, as in the rapidly oscillating Ap stars, when the pulsation axis does not lie along the star's rotation axis (i.e., the magnetic field axis in the Ap star case). Then the rotation of the star brings progressively different regions of the surface into view, altering the observed phase of the oscillations - and their amplitude. So again, this mechanism is ruled out by the constant amplitude we observe the $615 \mathrm{~s}$ period to have, because changes in the angle between
TABLE 3

PARAMETERS OF FIT

\begin{tabular}{cc}
\hline \hline Parameter & Value \\
\hline$a \sin i \ldots \ldots \ldots$. & $0.21 \pm 0.01$ A.U. \\
$e \ldots \ldots \ldots \ldots \ldots$. & $0.65 \pm 0.08$ \\
$\omega \ldots \ldots \ldots \ldots \ldots$. & $77^{\circ} \pm 9^{\circ}$ \\
$T_{0} \ldots \ldots \ldots \ldots \ldots$. & $1.7 \pm 1.4 \times 10^{5} \mathrm{~s}$ \\
$P \ldots \ldots \ldots \ldots \ldots$. & $9.4 \pm 1.1 \times 10^{6} \mathrm{~s}$ \\
\hline
\end{tabular}

the pulsation axis and the line-of-sight also produce amplitude modulations (see Kurtz 1982, 1988 and references therein for a detailed discussion of this point concerning the rapidly oscillating Ap stars, and the book by Unno et al. 1989 for a general discussion of variations in amplitude with inclination angle to the pulsation axis).

\section{THE BINARY MODEL}

We were therefore led to an extrinsic explanation for the phase variations: light travel time effects due to reflex orbital motion about an unseen companion. To match the phase variations observed, the orbit would have to be one of considerable eccentricity, and to be a plausible model the phase variations had to match, within observational error, the pattern of phase changes expected from objects in Keplerian orbits around their center of mass.

This model provides, in fact, an excellent fit to the timings. The fit we obtained using a generalized, nonlinear leastsquares routine called GaussFit, developed by Jefferies et al. (1988) is plotted against the data as a solid line in Figure 6; its parameters are shown in Table 3. A formal chi-squared test, based on the observed distribution of points around the fitting line, yields a (reduced) value $\chi^{2}=1.12$.

The inset in Figure 6 shows the same data and theoretical fit on a more condensed scale, where the large eccentricity can be better appreciated. Although the data look qualitatively similar to velocity curves typical of binaries with highly elliptical orbits, the time-lag $(O-C)$ diagram is actually the integral of the velocity curve: the quantity changing with time is the line-of-sight distance to G29-38.

The reflex motion model could be tested directly. It predicted that radial velocity variations, corresponding to the reflex orbital motion of the visible object, should exceed $50 \mathrm{~km} \mathrm{~s}^{-1}$, and should follow a very distinctive pattern, as shown in Figure 7. Published observations of G29-38 obtained by Liebert, Saffer, and Pilachowski (1989) had internal errors small enough ( $1 \sigma$ was between 6 and $13 \mathrm{~km} \mathrm{~s}^{-1}$ ) to have seen the velocity variations in the reflex motion our model predicts; unfortunately they had only three runs, with only 17 days between the first and the last. The velocity difference they found was smaller than their errors, as might be expected from their sampling interval.

The reflex motion model fitted the observed data so well that we became convinced that it was the correct explanation for the phase variations, and we solicited confirmation from our colleagues equipped to make the exacting radial velocity measurements required. We were confident the predicted velocity changes would be observed; nature could not be so perverse, we reasoned, as to provide so good a fit to a model that was wrong.

We were mistaken. Graham et al. (1990) measured the sharp absorption core of the $\mathrm{H} \alpha$ line in G29-38 and were unable to 


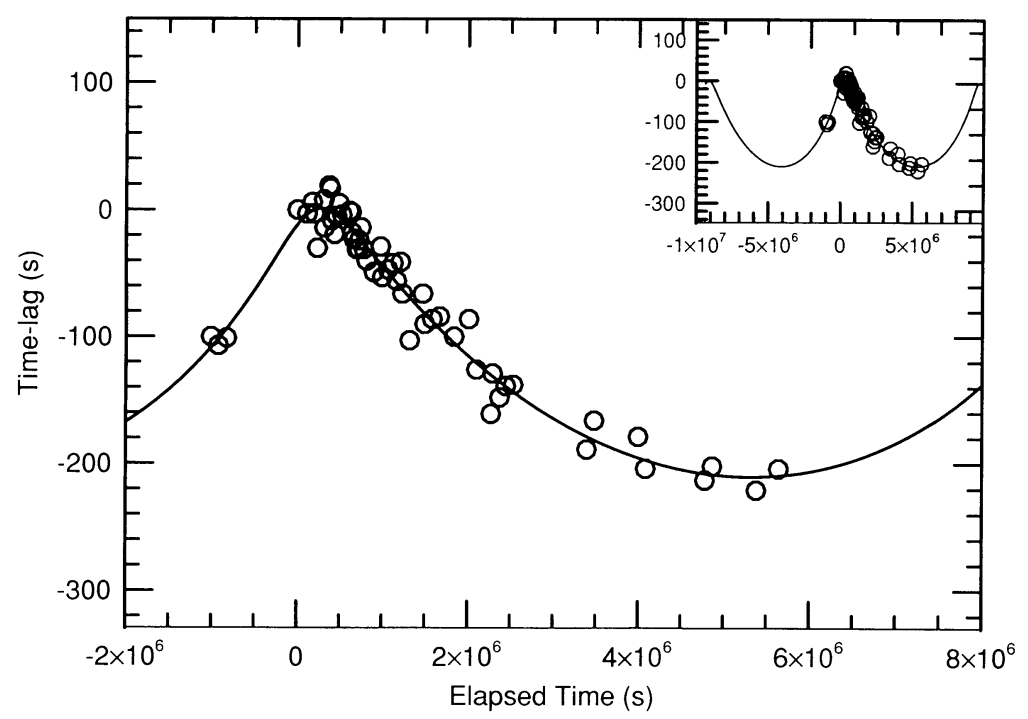

Fig. 6.-The time-lag $(O-C)$ diagram. Individual values (open circles) are shown, with the best fitting curve of orbital reflex motion superposed (solid line). The inset shows the same curve on a more condensed scale.

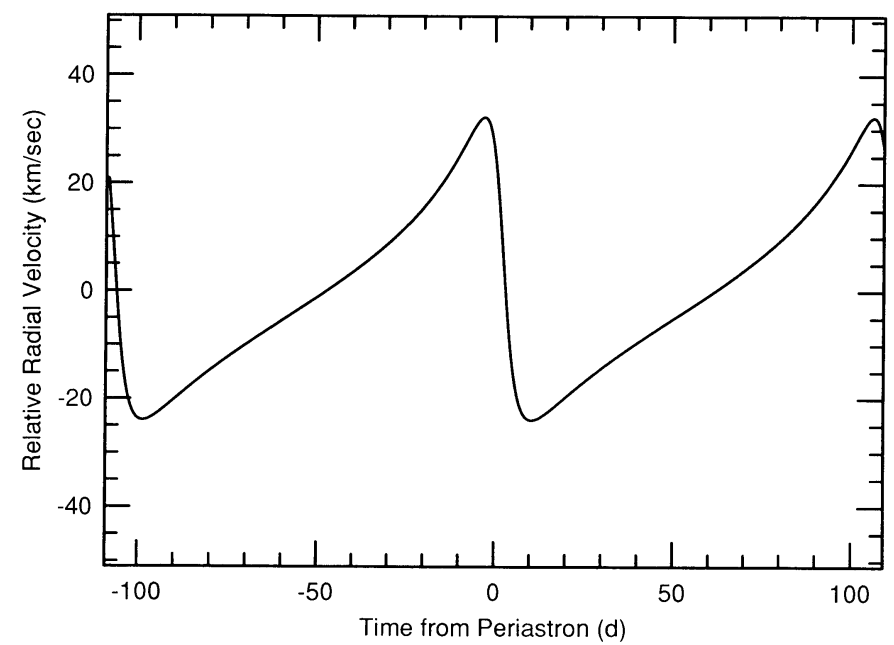

FIG. 7.-The computed radial velocity curve for the reflex orbital motion, derived from the time-lag $(O-C)$ curve in Fig. 5 .

find any variations in radial velocity, to within an uncertainty of $\pm 10 \mathrm{~km} \mathrm{~s}^{-1}$. Our preliminary analysis of single-site photometry taken during the $1989-1990$ observing season seems to bear out this result. A pulsation at $283.78 \mathrm{~s}$ shows the ampli- tude stability required to act as a good clock, but fails to display the variations in phase predicted by the binary model.

\section{SUMMARY AND CONCLUSIONS}

We have measured a phase variation in the time of arrival of optical pulsations from the $615 \mathrm{~s}$ periodicity in the DAV variable G29-38. The effect is far from subtle: we see a phase change amounting to $\sim 200 \mathrm{~s}$, almost a third of the period, but with no corresponding change in pulsation amplitude. The shape of the variation can be fit, to within measurement accuracy, by a model involving a nonluminous companion in orbit around the white dwarf. However, predictions of a corresponding, repeating variation in the phase of other pulsations, and in the radial velocity of the white dwarf, are not observed.

This phase variation is not readily explained by any intrinsic pulsation process we can identify. Its presence therefore provides both a mystery and a challenge to the current theory of non-radial stellar oscillations, whose linear realization cannot explain the effects observed. Some unknown nonlinear process may well be calling itself to our attention.

We are grateful for support from NSERC Canada, CNRS France, the National Science Foundation grants AST 8552456, AST 86-00507, AST 87-12249, AST 88-13572, NASA grant NGT-50210 and from the National Geographic Society grant 3547-87. We thank an anonymous referee for his suggestions concerning the presentation of this material.

\section{REFERENCES}

Buchler, J. R. 1988, Multimode Stellar Pulsations (Kultura, Budapest : Konkoly Observatory), p. 71.

Cox, J. P. 1980, Theory of Stellar Pulsation (Princeton: Princeton University Press).

Dziembowski, W. 1988, Multimode Stellar Pulsations (Kultura, Budapest: Konkoly Observatory), p. 127

Graham, J. R., McCarthy, J. K., Reid, I. N. and Rich, R. M. 1990, preprint.

Greenstein, J. L. 1988, A.J., 95, 1494

Jeffries, W. H., Fitzpatrick, J. E., McArthur, B. E., and McCartney, J. E. 1988, GaussFit: A System for Least Squares and Robust Estimation USER'S MANU AL (Austin: University of Texas, Dept. of Astronomy).

Kawaler, S. D. 1988, in IAU Symposium 123, Advances in Helio- and Asteroseismology, ed. J. Christiansen-Dalsgaard and S. Fransden (Dordrecht: Reidel), p. 329.
Kepler, S. O., Robinson, E. L., and Nather, R. E. 1983, Ap. J., 271, 744

Kepler, S. O., Robinson, E. L., Nather, R. E., and McGraw, J. T. 1982, Ap. J., 254, 676

Kepler, S. O., Vauclair, G., Nather, R. E., Winget, D. E., and Robinson, E. L. 1989, in IAU Colloquium 114, White Dwarfs, ed. G. Wegner (Berlin: Springer-Verlag), p. 341

Kurtz, D. W. 1982, M.N.R.A.S., $200,807$.

. 1988, Multimode Stellar Pulsations (Kultura, Budapest: Konkoly Observatory), p. 107.

Lester, D. 1989, private communication.

Liebert, J., Saffer, R. A., and Pilachowski, C. A. 1989, A.J.,97, 182.

McGraw, J. T., and Robinson, E. L. 1975, Ap. J.(Letters), 200 , L89.

Nather, R. E. 1989, in IAU Colloquium 114, White Dwarfs, ed. G. Wegner, (Berlin: Spriner-Verlag), p. 109. 
O'Donoghue, D., and Warner, B. 1987, M.N.R.A.S., 228, 949.

Stover, R. J., Hesser, J. E., Lasker, B. M., Nather, R. E., and Robinson, E. L. 1980, Ap. J., 240, 865 .

Tokunaga, A. T., Hodapp, K.-W., Becklin, E. E., Cruikshank, D. P., Rigler, M.

Toomey, D., Brown, R. H., and Zuckerman, B. 1988, Ap. J. (Letters), 332, L71.

Unno, W., Osaki, Y., Ando, H., Saio, H., and Shibihashi, H. 1989, Nonradial Oscillations of Stars, 2d ed., (Tokyo: University of Tokyo Press).
Winget, D. E. 1988, in IAU Symposium 123, Advances in Helio- and Asteroseismology, ed. J. Christiansen-Dalsgaard and S. Fransden (Dordrecht: Reidel), p. 305.

Zuckerman, B., and Becklin, E. E. 1987a, Ap. J.(Letters), 319, L99.

. 1987b, Nature, 330, 138.

N. ACHILleos: Department of Mathematics, Australian National University, Canberra, Australia

B. N. Ashoka, T. M. K. Marar, and S. Seetha: Technical Physics Division, ISRO Satellite Centre, Airport Road, Bangalore 560 017, India

T. AugusteIJn: European Southern Observatory, La Silla, Chile

P. A. Bradley, C. F. Claver, J. C. Clemens, S. J. Kleinman, R. E. Nather, J. Provencal, E. L. Robinson, D. E. Winget, and M. A. WooD: Department of Astronomy, University of Texas, Austin, TX 78712,

M. Chevreton: Observatoire de Paris-Meudon, F-92195 Meudon, Principal Cedex, France

G. Fontaine: Departement de Physique, Université de Montréal, C.P. 6128, Succ. A. Montréal, Quebec, Canada H3C 3J7

A. D. GraUER: Department of Physics and Astronomy, University of Arkansas, 2801 S. University Avenue, Little Rock, AR 72204

C. J. HANSEN : Joint Institute for Laboratory Astrophysics, University of Colorado, Box 448, Boulder, CO 80309

B. P. Hine: M.S. 244-4, NASA Ames Research Center, Moffett Field, CA 94035

A. KanaAn and S. O. KePler: Instituto de Fisica, Universidade Federal do Rio Grande do Sul, 90049 Porto Alegre-RS, Brasil

D. W. Kurtz, P. Martinez, D. O’Donoghue, and B. Warner: Department of Astronomy, University of Cape Town, Rondebosh 7700, Cape Province, South Africa

JAMES LIEBERT: University of Arizona, Steward Observatory, Tucson, AZ 85721

J. VAN PARAdIJS: Universiteit van Amsterdam, Faculteit der Wiskunde en Natuurwetenschappen, Roetersstreat 151018 WB, Amsterdam, The Netherlands

G. VAUClaIR: Observatoire Midi-Pyrenees, 14 Avenue E. Belin, 31400 Toulouse, France 